\title{
A simple and sensitive fluorescent probe for specific detection of cysteine
}

\author{
YAN-FEI KANG*, HAI-XIA QIAO, YA-LI MENG, ZHEN-HUI XIN, LI-PING GE, \\ JIN-NAN ZHENG and YI-NA WANG \\ College of Laboratory Medicine, Hebei North University, 11 Diamond Street South, Zhangjiakou 075000, \\ Hebei Province, People's Republic of China \\ E-mail: kangyanfei172@163.com
}

MS received 7 May 2017; revised 1 June 2017; accepted 13 June 2017

\begin{abstract}
A fluorescent probe, with simplicity of structure and convenience of synthesis, is capable of detecting cysteine over other biothiols, such as homocysteine and glutathione. The detection limit of 60 nM and 190-fold increase in fluorescence intensity offer advantage for detection of Cys in biological systems. Furthermore, the probe 1 could be applied in bioimaging.
\end{abstract}

Keywords. Fluorescence probe; cysteine; selectivity; bioimaging.

\section{Introduction}

Bio-thiols such as cysteine (Cys), homocysteine (Hcy), and glutathione (GSH) play pivotal roles in various biological processes including protein synthesis, detoxification, metabolism, signal transduction and gene regulation. ${ }^{1,2}$ In addition, these bio-thiols are essential in regulating of the redox state of proteins and in protein structure. More importantly, these species are involved in many crucial functions, for example, cysteine (Cys) and homocysteine (Hcy) are related to variety of human diseases like neurotoxicity, skin lesions, edema, muscle weakness, lethargy, slow growth, hair depigmentation, ${ }^{3}$ cardiovascular ${ }^{4}$ and Alzheimer's diseases. ${ }^{5}$ Similarly, glutathione (GSH) is the most abundant intracellular non-protein thiol and is considered to be an indicator of oxidative stress. ${ }^{6,7}$ As a consequence, detection of these low molecular weight thiols is important and timely. Therefore, a great many of detection methods were developed, but the fluorescence probe detection obtained considerable attention on the basis of its simplicity, efficiency, low detection limits and ease of handling. ${ }^{8-10} \mathrm{Up}$ to now, the fluorescence probes were designed and synthesized mainly according to the different chemical reaction mechanisms, such as Michael addition, cyclization, conjugate

\footnotetext{
*For correspondence
}

addition-cyclization, cleavage reaction by thiols, thiolhalogen nucleophilic substitution, disulfide exchange, and others. ${ }^{4,11-16}$ However, selective detection of Cys is difficult in view of structural similarity of Cys, Hcy and GSH. Thus, development of probes to selectively detect Cys also remains challenging for the scientific community.

Among these approaches, the selective detection of Cys based on the conjugate addition-cyclization of Cys with acrylates has proven to be specifically effective, this strategy has attracted broad attention. ${ }^{17-24}$ Moreover, coumarin, a well-known fluorophore, exhibits low cytotoxicity and possesses favorable photophysical properties such as a large Stokes shift, visible excitation and emission wavelengths, good photostability, reasonable fluorescent quantum yield and easy synthesis. ${ }^{25-27}$ Thus, in this work, we designed and synthesized fluorescent probe 1 (ethyl 7-(acryloyloxy)-2-oxo- $2 H$-chromene-3carboxylate) with a latent coumarin fluorophore and a receptor of acrylate. We envision that the probe 1 is capable of detecting selectively Cys over Hcy and GSH.

\section{Experimental}

Unless otherwise noted, materials were purchased from commercial suppliers and used without further purification. All the solvents were purified and dried according to

Electronic supplementary material: The online version of this article (doi:10.1007/s12039-017-1326-y) contains supplementary material, which is available to authorized users. 


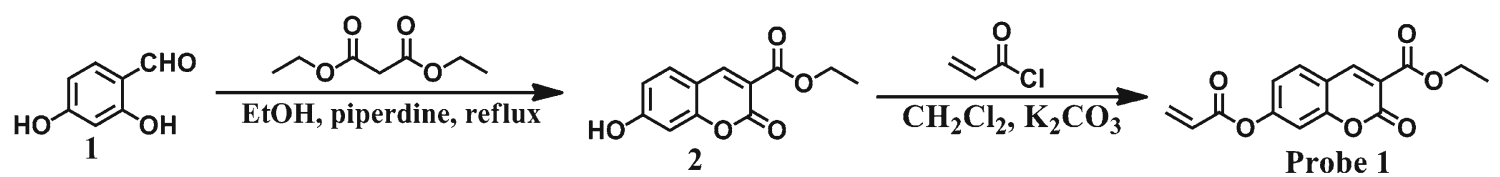

Scheme 1. Synthesis of probe 1.
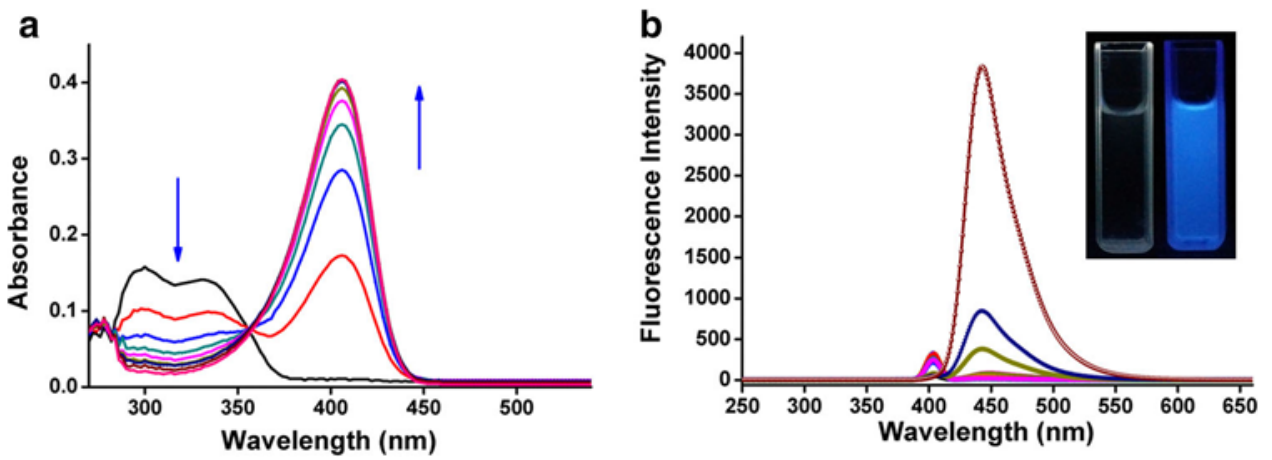

Figure 1. (a) With addition of Cys $(100 \mu \mathrm{M})$, UV-Vis absorption spectra of probe 1 $(10 \mu \mathrm{M})$ with increase of incubation time from 0 to $15 \mathrm{~min}$. (b) Fluorescence spectra of probe $1(10 \mu \mathrm{M})$ in the presence of various analytes $(100 \mu \mathrm{M})$ in an aqueous PBS buffer $(\mathrm{pH}$ 7.4, containing $20 \%$ acetonitrile); $\lambda_{\mathrm{ex}}=406 \mathrm{~nm}$. Inset shows a picture of probe 1 without and with Cys under ultraviolet radiation.

general methods. Synthesis and Characterization of probe 1, and other experimental details are given in Supporting Information.

\section{Results and Discussion}

Keeping the following considerations in mind, namely, acrylates can recognize Cys based on conjugate addition-cyclization and coumarin is a well-known fluorophore, probe 1 was synthesized (Scheme 1, for details and characterization see the Supporting Information) and structure confirmed by NMR and HRMS (ESI). Initially, we explored the UV-Vis absorption spectrum of probe $1(10 \mu \mathrm{M})$ in phosphate buffer (PBS pH 7.4, containing 20\% acetonitrile). Probe 1 had obvious absorption peaks at 300 and $335 \mathrm{~nm}$. However, with addition of Cys $(100 \mu \mathrm{M})$, the absorption bands at 300 and 335 gradually decreased with concomitant growth of a new band at $406 \mathrm{~nm}$ (Figure 1a). Thus, 406 $\mathrm{nm}$ was identified as excitation spectrum wavelength. Subsequently, the fluorescence intensity of probe 1 was screened with the different amino acids (L-Cys, Hcy, GSH, Arg, Asp, Tyr, Pro, Lys, Gly, Ala, Val, Phe, Thr,

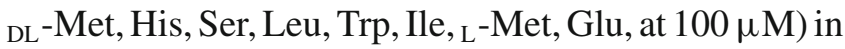
phosphate buffer (PBS pH 7.4, containing 20\% acetonitrile) (Figure 1b). Interestingly, a remarkable 190-fold enhancement in fluorescence intensity was observed with the addition of Cys and the solution, after $5 \mathrm{~min}$ incubation time, showed strong blue fluorescence with an excitation at $406 \mathrm{~nm}$. However, other amino acids did not cause distinct change of fluorescence intensity; Hcy and GSH showed slight interference, however. Therefore, the probe 1 could selectively detect Cys level.

In order to explore the effects of other analytes for detection of Cys, we carried out the competiton experiment (Figure S1 in Supplementary Information). The results indicated that fluorescence intensity of probe 1 for Cys did not change clearly in the presence of other amino acids or metal ions, even Hcy and GSH. Moreover, there is only $20 \%$ decrease in fluorescence intensity of probe 1 toward Cys in the presence of GSH /Hcy $(200 \mu \mathrm{M})$. Thus, these experimental results further showed that the probe $\mathbf{1}$ could distinguish Cys from other amino acids. In addition, the fluorescence intensity was enhanced gradually with the increase of Cys content from 1 equiv. to 10 equiv. (Figure 2a). Subsequently, we further investigated the properties of the probe 1. To investigate the fluorescence spectral response of probe $\mathbf{1}$ for Cys, we extended the action time of Cys to $20 \mathrm{~min}$. The result displayed that the increase of fluorescence intensity reached the plateau stage after $10 \mathrm{~min}$ (Figure 2b). Thus, this response is faster than those reported previously. ${ }^{24}$ Furthermore, the fluorescence intensity of probel toward Hcy/GSH increased with extension of response time. However, this enhancement is slower and weaker than of probe 1 toward Cys. Meanwhile, the probe $\mathbf{1}$ did not induce any obvious change by itself, which meant the probe $\mathbf{1}$ was stable under this reaction condition. On the same basis, 

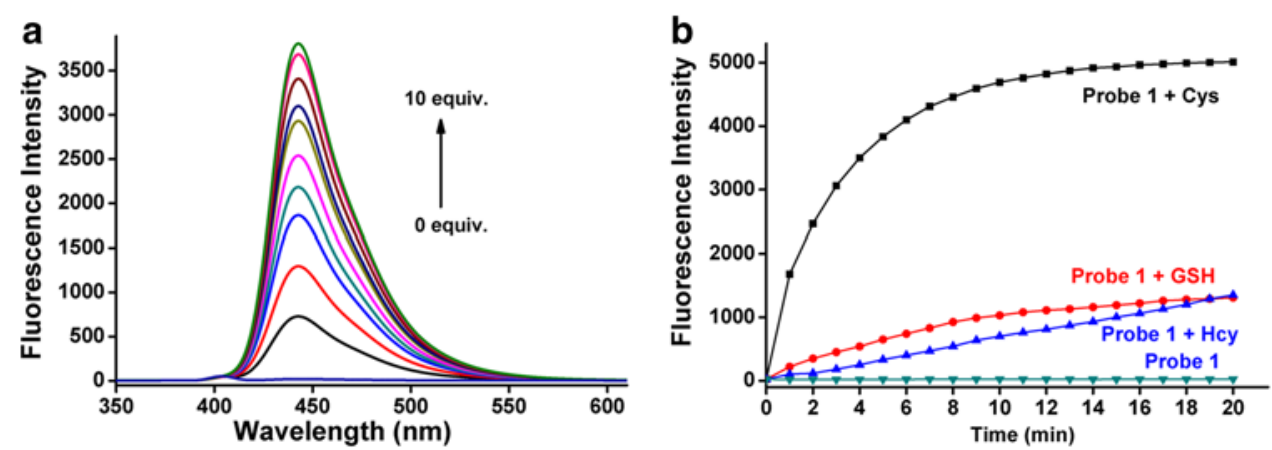

Figure 2. (a) Emission spectra of probe $1(10 \mu \mathrm{M})$ with increase of Cys concentration (from 0 to 10 equiv.) after incubation of $5 \mathrm{~min}$ at room temperature. (b) Time-dependent fluorescence intensity of probe $1(10 \mu \mathrm{M})$ in the presence and absence of Cys (10 equiv.), $\lambda_{\mathrm{ex}}=406 \mathrm{~nm}$.<smiles>C=CC(=O)Oc1ccc2cc(C(=O)OCC)c(=O)oc2c1</smiles>

Probe 1

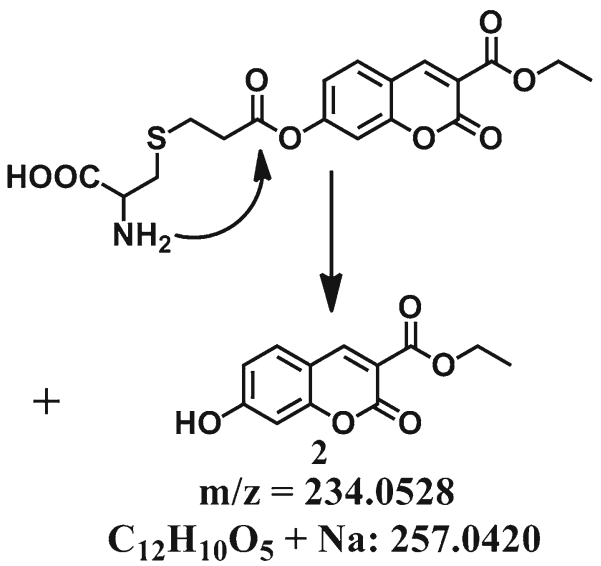

Scheme 2. Proposed reaction mechanism of probe 1 with Cys.

the detection limit ( $3 \mathrm{~s} / \mathrm{m}, \mathrm{n}=20$ ) of Cys was evaluated to be $60 \mathrm{nM}$ (Figure S2 in Supplementary Information). ${ }^{28,29}$ More importantly, the fluorescence intensity of probe 1 was weak in $\mathrm{pH}$ 3.0-9.0; however, with addition of Cys, the fluorescence intensity was enhanced obviously in $\mathrm{pH}$ 6.0-9.0. Thus, probe $\mathbf{1}$ is capable of detecting selectively Cys level under the conditions of physiological pH (Figure S3 in Supplementary Information).

We further investigated the reaction mechanism between probe 1 and Cys. Cys $(100 \mu \mathrm{M})$ was added to the solution of probe $1(10 \mu \mathrm{M})$, and we completed high resolution mass spectrometry analysis of the mixture. The peak at $\mathrm{m} / \mathrm{z}=257.0421$, corresponding to product $2(\mathrm{M}+\mathrm{Na})$ was clearly discovered (Figure S4 in Supplementary Information). Therefore, we propose a probable reaction mechanism (Scheme 2). The initial conjugate addition of Cys to acrylate moiety and subsequent intramolecular cyclization afforded the final fluorescent compound, which caused increase of fluorescence intensity. Meanwhile, we measured the pseudo- first-order rate constant $k^{\prime}$ at room temperature. The pseudo-first-order rate constants for Cys, Hcy, and GSH were measured as $k^{\prime}=1.2 \times 10^{-2} \mathrm{~s}^{-1}, 1.0 \times 10^{-3} \mathrm{~s}^{-1}$, $1.5 \times 10^{-3} \mathrm{~s}^{-1}$, respectively (Figure S5 in Supplementary Information). Thus, Cys induced a rapid cyclization reaction of a stable seven-membered ring, which is kinetically favorable. This difference of reaction rate made that the probe $\mathbf{1}$ could selectively and quickly detect Cys over Hcy/GSH.

Lastly, we studied the application of probe $\mathbf{1}$ in livecell imaging. Before the cell imaging was investigated, we first measured the cytotoxic activity of probe 1 by MTT method. ${ }^{30}$ Interestingly, the results displayed that the probe $1(20 \mu \mathrm{M})$ did not induce apparent cell death within $24 \mathrm{~h}$ of incubation time (Figure 6 in Supplementary Information). Subsequently, we recorded the bioimaging of probe 1 (Figure 3). When the HeLa cells were incubated with probe $1(10 \mu \mathrm{M})$ for $1 \mathrm{~h}$, they became blue fluorescent. More importantly, when the cells were pretreated with thiol scavenger (N-ethyl maleimide, NEM, $1 \mathrm{mM}$ ) for $30 \mathrm{~min}$, the blue fluores- 


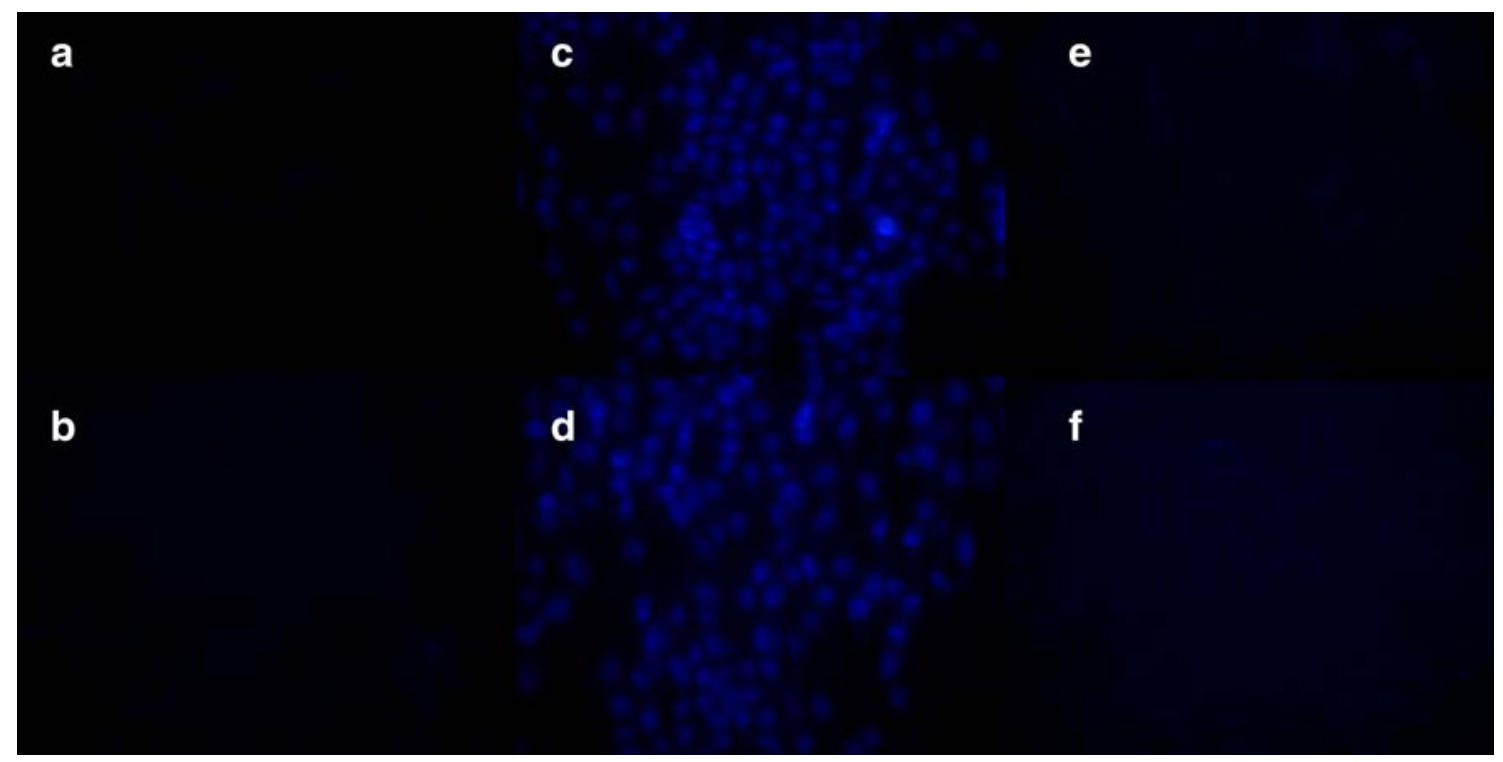

Figure 3. Bioimaging of HeLa cells. (A) Blank; (B) Cells after incubation with DMSO for 1 h; (C, D) Cells after incubation with probe $\mathbf{1}(10 \mu \mathrm{M})$ for $1 \mathrm{~h}$; (E, F) cells which were pre-incubated with NEM (1 mM) for $30 \mathrm{~min}$. $\lambda_{\mathrm{ex}}=406 \mathrm{~nm}$.

cence did not appear with cells incubated by probe $\mathbf{1}$. Therefore, probe $\mathbf{1}$ can be applied for sensing Cys in living cells.

\section{Conclusions}

We designed and synthesized the fluorescent probe 1, which displayed a highly selective and sensitive response to Cys over Hcy/GSH in the tests. Moreover, the 190-fold increase in fluorescence intensity, and detection limit of $60 \mathrm{nM}$ offered a powerful method for practical detection of Cys in biological systems. Also, the probe $\mathbf{1}$ could be applied in bioimaging. Thus, we believe that this new probe will be of great benefit for gerater understanding of the roles of Cys in the biological systems.

\section{Supplementary Information (SI)}

All additional information pertaining to the characterization of compounds using ${ }^{1} \mathrm{H}$ NMR,${ }^{13} \mathrm{C}$ MNR spectra, and HRMS, and experimental methods are given in the Supporting Information, which is available at www.ias. ac.in/chemsci.

\section{Acknowledgements}

This work was supported by the Natural Science Foundation of Hebei Province (No. B2016405026), Young Elitist Foundation of Hebei Province (No. BJ2016003), Guidance projects of Science and Technology of Zhangjiakou City (No.
1621122H), and Guide Projects of Department of Education of Hebei Province (No. Z2017026).

\section{References}

1. Kemp M, Go Y M and Jones D P 2008 Nonequilibrium thermodynamics of thiol/disulfide redox systems: a perspective on redox systems biology Free Radical Biol. Med. 44921

2. Ball R O, Courtney-Martin G and Pencharz P B 2006 The in vivo sparing of methionine by cysteine in sulfur amino acid requirements in animal models and adult humans $J$. Nutr. $1361682 \mathrm{~S}$

3. Shahrokhian S 2001 Lead phthalocyanine as a selective carrier for preparation of a cysteine-selective electrode Anal. Chem. 735972

4. Jung H S, Chen X, Kim J S and Yoon J 2013 Recent progress in luminescent and colorimetric chemosensors for detection of thiols Chem. Soc. Rev. 426019

5. Seshadri S, Beiser A, Selhub J, Jacques P F, Rosenberg I H, D'Agostino R B, Wilson P W F and Wolf P A 2002 Plasma Homocysteine as a Risk Factor for Dementia and Alzheimer's Disease N. Engl. J. Med. 346476

6. Pastore A, Federici G, Bertini E and Ptemonte F 2003 Analysis of glutathione: implication in redox and detoxification Clin. Chim. Acta 33319

7. Sabelle S, Renard P Y, Pecorella K, Suzzoni-Dezard S, Creminon C, Grassi J and Mioskowski C 2002 Design and synthesis of chemiluminescent probes for the detection of cholinesterase activity J. Am. Chem. Soc. 124 4874

8. Wu J, Liu W, Ge J, Zhang H and Wang P 2011 New sensing mechanisms for design of fluorescent chemosensors emerging in recent years Chem. Soc. Rev. 403483

9. Schaeferling M 2012 The art of fluorescence imaging with chemical sensors Angew. Chem. Int. Ed. $\mathbf{5 1} 3532$ 
10. Kobayashi H, Ogawa M, Alford R, Choyke P L and Urano Y 2010 New Strategies for Fluorescent Probe Design in Medical Diagnostic Imaging Chem. Rev. 110 2620

11. Niu L-Y, Chen Y-Z, Zheng H-R, Wu L-Z, Tung C-H and Yang Q-Z 2015 Design strategies of fluorescent probes for selective detection among biothiols Chem. Soc. Rev. 446143

12. Zhou Y and Yoon J 2012 Recent progress in fluorescent and colorimetric chemosensors for detection of amino acids Chem. Soc. Rev. 4152

13. Chen X, Zhou Y, Peng X and Yoon J 2010 Fluorescent and colorimetric probes for detection of thiols Chem. Soc. Rev. 392120

14. Lee D, Kim G, Yin J and Yoon J 2015 An aryl-thioether substituted nitrobenzothiadiazole probe for the selective detection of cysteine and homocysteine Chem. Commun. 516518

15. Murale D P, Kim H, Choi W S and Churchill D G 2013 Highly Fluorescent and Specific Molecular Probing of (Homo)Cysteine or Superoxide: Biothiol Detection Confirmed in Living Neuronal Cells Org. Lett. 153630

16. Murale D P, Kim H, Choi W S, Kima Y and Churchill D G 2014 Extremely selective fluorescence detection of cysteine or superoxide with aliphatic ester hydrolysis $R S C$ Adv. 446513

17. Yang X, Guo Y and Strongin R M 2011 Conjugate addition/cyclization sequence enables selective and simultaneous fluorescence detection of cysteine and homocysteine Angew. Chem. Int. Ed. 5010690

18. Niu W, Guo L, Li Y, Shuang S, Dong C and Wong M S 2016 Highly selective two-photon fluorescent probe for ratiometric sensing and imaging cysteine in mitochondria Anal. Chem. 881908

19. Kang Y-F, Qiao H-X, Meng Y-L, Cui S-J, Han Y-J, Wu Z-Y, Wu J, Jia X-H, Zhang X-L and Dai M-Y 2016 Rapid and selective detection of cysteine over homocysteine and glutathione by a simple and effective coumarin-based fluorescent probe RSC Adv. 694866

20. Han C, Yang H, Chen M, Su Q, Feng W and Li F 2015 Mitochondria-targeted near-infrared fluorescent off-on probe for selective detection of cysteine in living cells and in vivo ACS Appl. Mater. Interfaces 727968

21. Liu Y, Yu D, Ding S, Xiao Q, Guo J and Feng G 2014 Rapid and ratiometric fluorescent detection of cysteine with high selectivity and sensitivity by a simple and readily available probe ACS Appl. Mater. Interfaces 617543

22. Liu B, Wang J, Zhang G, Bai R and Pang Y 2014 Flavonebased ESIPT ratiometric chemodosimeter for detection of cysteine in living cells ACS Appl. Mater. Interfaces 6 4402

23. Zhu B, Guo B, Zhao Y, Zhang B and Du B 2014 A highly sensitive ratiometric fluorescent probe with a large emission shift for imaging endogenous cysteine in living cells Biosens. Bioelectron. 5572

24. Dai X, Wu Q-H, Wang P-C, Tian J, Xu Y, Wang S-Q, Miao J-Y and Zhao B-X 2014 A simple and effective coumarin-based fluorescent probe for cysteine Biosens. Bioelectron. 5935

25. Chen J, Liu W, Zhou B, Niu G, Zhang H, Wu J, Wang Y, Ju W and Wang P 2013 Coumarin- and rhodaminefused deep red fluorescent dyes: synthesis, photophysical properties, and bioimaging in vitro J. Org. Chem. $\mathbf{7 8 6 1 2 1}$

26. Lim N C and Brückner C 2004 DPA-substituted coumarins as chemosensors for zinc(II): modulation of the chemosensory characteristics by variation of the position of the chelate on the coumarin Chem. Commun. 1094

27. Trenor S R, Shultz A R, Love B J and Long T E 2004 Coumarins in polymers: from light harvesting to photocross-linkable tissue scaffolds Chem. Rev. 1043059

28. MacDougall D and Crummett W B 1980 Guidelines for data acquisition and data quality evaluation in environmental chemistry Anal. Chem. 522242

29. Hong K-H, Lim S-Y, Lun M-Y, Lim J-W, Woo JH, Kwon H and Kim H-J 2013 Selective detection of cysteine over homocysteine and glutathione by a bis(bromoacetyl)fluorescein probe Tetrahedron Lett. 54 3003

30. Hussain R F, Nouri A M E and Oliver R T 1993 A new approach for measurement of cytotoxicity using colorimetric assay J. Immunol. Methods 16089 